worse prognosis hazard ratio $=3.6(95 \% \mathrm{CI}=1.24-10.44, \mathrm{p}=$ 0.00184). Although the NT-pro BNP correlated with LVEF its association with outcome was independent of this.

Conclusions In a prospective mixed cohort of patients with AS undergoing investigations for consideration of aortic valve intervention, even a mild elevation in NT-pro BNP is associated with worse short term outcome. This can be taken into consideration regarding timing of intervention especially in the asymptomatic population.

\section{Basic Science}

\section{A CRUCIAL ROLE OF NOX2-DERIVED REACTIVE OXYGEN SPECIES IN AGEING-ASSOCIATED METABOLIC DISORDERS AND BRAIN OXIDATIVE DAMAGE}

Li Geng", Jian-Mei Li. University of Reading; *Presenting Author

\subsection{6/heartinl-2016-309890.159}

Ageing has been recognised to be a major risk factor for the development of cardiovascular and neurodegenerative diseases and growing evidence suggests a role for oxidative stress. A Nox2-containing NADPH oxidase has been reported to be a major source of reactive oxygen species (ROS) generation in the vascular system and in the brain. However, the role of Nox2 enzyme in ageing-related metabolic disorders and vascular neurodegeneration remains unclear. In this study, we used age-matched wild-type (WT) and Nox2-deficient (Nox2 $2^{-/-}$) mice on a C57BL/6 background at young (3-4 month) and ageing (20-24 month) to investigate the role of Nox2 in ageing-related oxidative stress, metabolic disorders and cerebral vascular dysfunction. There was an ageing-related increase in blood pressure in WT mice (126 $\mathrm{mmHg}$ for young and 148 $\mathrm{mmHg}$ for ageing) $(\mathrm{P}<0.05)$; however the blood pressure was well maintained without significant change in Nox $2^{-/-}$ageing mice. Compared to young WT mice, WT ageing mice had significantly high levels of fasting serum insulin and this was accompanied with delayed clearance of glucose $(\mathrm{P}<0.05)$ indicating insulin resistance. In contrast, there was no indication of insulin resistance for $\mathrm{Nox}^{-/-}$ageing mice. We then examined ageing-related brain oxidative stress. Compared to WT young mice, there were significant increases $(2.7 \pm 0.7$ folds) in the levels of ROS production by WT ageing brain tissue homogenates as detected by lucigenin-chemiluminescence and DHE fluorescence. Increased ROS production in WT ageing brain was accompanied by a significant increase $(1.8 \pm$ 0.3 folds) in the Nox2 expression detected mainly in the microglial cells (labelled by Iba-1) and decreases in brain capillaries (labelled by CD31) (2.4 \pm 0.8 folds) and neurons (labelled by Neuronal Nuclei) $(2.9 \pm 0.5$ folds) (all $\mathrm{P}<$ $0.05)$. Knockout Nox2 abolished ageing-associated increases in brain ROS production and reduced significantly the ageingrelated pathophysiological changes in the brain. In conclusion, Nox2-derived oxidative stress plays an important role in ageing-associated metabolic disorders and vascular neurodegeneration. Nox2-containg NADPH oxidase represents a valuable therapeutic target for oxidative stress-related diseases in ageing.

\section{0 MODULATION OF MYOCARDIAL ISCHAEMIC INJURY BY CARBON MONOXIDE RELEASING MOLECULE-A1 IN A PORCINE MODEL OF ACUTE REPERFUSION INFARCTION}

${ }^{1}$ Mabruka Alfaidi*, ${ }^{2}$ Matthew Hughes, ${ }^{2}$ Tooba Alizadeh, ${ }^{2}$ Helen Casbolt, ${ }^{2}$ Janet Chamberlain, ${ }^{2}$ Brian E Mann, ${ }^{2}$ Sheila E Francis, ${ }^{2}$ Javaid Iqbal, ${ }^{2} J u l i a n$ Gunn. ${ }^{1}$ The University of Sheffield; ${ }^{2}$ Sheffield University; *Presenting Author

\subsection{6/heartjnl-2016-309890.160}

Introduction Conventional pharmacological treatments for acute myocardial infarction (AMI), a life threatening complication of a sudden coronary occlusion, are limited by their efficiency and side effects. New therapeutic strategies are thus needed to improve outcomes. Carbon monoxide (CO) is cardio-protective at nanomolar concentrations. Carbon monoxidereleasing molecules (CORMs), capable of carrying and releasing controlled quantities of $\mathrm{CO}$ in cellular systems, are a promising therapeutic that overcome the limitations of $\mathrm{CO}$ gas. CORM-A1 is a water soluble and releases CO slowly through hydrolysis at physiological conditions. We investigated the efficacy and safety of CORM-A1 in reducing infarct size in a clinically relevant porcine model of re-perfused AMI.

Methods Male Yorkshire White pigs (25-33 kg) underwent a balloon-induced coronary occlusion at the middle segment of left anterior descending artery beyond the first diagonal branch for $60 \mathrm{~min}$. From $15 \mathrm{~min}$ post-occlusion, sodium borate (control) or CORM-A1 (4.27 mM, each) were infused over a period of $60 \mathrm{~min}$. Left ventricular (LV) function and blood pressure were assessed by cardiac catheterization. Cardiac biomarkers, hepatic and renal functions were compared between the groups. Seven days after AMI, animals were culled and in-situ double staining with Evans blue and 2,3,5-triphenyltetrazolium (TTC) performed to measure infarct size. Myocardial inflammation, proliferation and apoptosis were evaluated by immunohistochemistry, immunoblotting, and TUNEL assay.

Results CORM-A1 treated pigs had a reduced infarcted area and improved LV function, but no significant change in blood pressure, compared to controls. Infarct size was $35 \pm 7 \%$ of the area at risk (ischaemic area) in CORM-A1 pigs compared to $90 \pm 5 \%$ in controls ( $\mathrm{p}<0.0001, \mathrm{n}=3-8 /$ group). Myocardium from CORM-A1 treated animals had fewer TUNEL positive (30.5 \pm 4.7 vs. $46.2 \pm 6.6 \%, \mathrm{p}<0.05, \mathrm{n}=37)$, Ki67 positive $(7.7 \pm 2.3$ vs. $29.0 \pm 4.0 \%, \mathrm{p}<0.01, \mathrm{n}=3-$ $7)$ and inflammation positive cells $(4.3 \pm 1.8$ vs. $36.7 \pm$ $7.1 \%, \mathrm{p}<0.01, \mathrm{n}=3-7)$ in the infarcted regions, compared to controls. CORM-A1 infused animals also had significantly reduced (2-3-fold) neovascular formation (vWF staining) in the infarcted areas compared to controls $(22.0 \pm 3.6$ vs. 53.3 $\pm 8.9 \%, \mathrm{p}<0.01, \mathrm{n}=3-7)$. A similar pattern was seen in the ischaemic areas. These changes were associated with a down-regulation of HIF-1a expression in the myocardium of CORM-A1 treated animals.

Conclusions Our data suggest CORM-A1 as a key modulator of myocardial repair following re-perfused AMI injury. Injury is reduced in CORM-A1 treated animals by reducing inflammation, proliferation and cell death whilst maintaining healthy repair via neovascularisation. This study suggests the development of CORM-A1 as a potential new therapeutic for treatment of patients with AMI and warrants further clinical studies. 